\title{
PRESERVATION OF INTERCOSTAL ARTERIES DURING THORACOABDOMINAL AORTIC ANEURYSM SURGERY: A RETROSPECTIVE STUDY
}

Scott D. Ross, MD

Irving L. Kron, MD

Patrick E. Parrino, MD

Kimberly S. Shockey, MS

John A. Kern, MD

Curtis G. Tribble, MD
Objective: The purpose of this article was to examine the influence of reimplantation of patent intercostal and lumbar arteries on the incidence of postoperative paraplegia/paraparesis in patients undergoing clamp-and-sew surgical repair of thoracoabdominal aortic aneurysms. Methods: Data from January 1987 through December 1997 were retrospectively collected on 132 patients. Ninety-one patients in group I underwent aneurysm repairs before January 1995 and did not undergo intercostal artery reimplantation. Group II included the more recent 41 patients who had vessels between the eighth thoracic intercostal and the second lumbar arteries reimplanted to the graft or preserved at the aortic anastomoses. Results: The operative mortality rate was $13.2 \%(12 / 91)$ in group $I$ and $4.9 \%(2 / 41)$ in group II $(P=.22)$. The incidence of postoperative paraplegia was significantly lower in the more recent cohort of patients $(8.8 \%$ [8/91] in group I vs $0 \%$ [0/41] in group II, $P=.05)$. The overall rate of spinal cord dysfunction was lowered from $9.9 \%(9 / 91)$ in group I to $2.4 \%(1 / 41)$ in group II $(P=.17)$. However, a multivariable logistic regression analysis identified only aneurysm extent (Crawford type I and type II) as a predictor of less postoperative spinal cord injury $(P=.08)$. The average aortic crossclamp time in group I was $30.3 \pm 11.5$ (SD) minutes, and the time of aortic occlusion in group II was not significantly prolonged, with an average crossclamp time of $31.0 \pm 21.0$ (SD) minutes $(P=.88)$. Conclusions: An aggressive approach to maintain intercostal artery patency during clamp-and-sew repair of thoracoabdominal aortic aneurysms may effectively lower the incidence of spinal cord injury without prolonging aortic crossclamp time. (J Thorac Cardiovasc Surg 1999;118:17-25)
$T^{\text {he }}$ he surgical repair of aneurysms of the thoracoabdominal aorta has undergone considerable advancements over the past several decades. Despite many surgical successes, the threat of spinal cord ischemia and postoperative paraplegia remains an unsolved problem

From the Department of Surgery, Division of Thoracic and Cardiovascular Surgery, University of Virginia Health Sciences Center, Charlottesville, Va.

Read at the Twenty-fourth Annual Meeting of The Western Thoracic Surgical Association, Whistler, British Columbia, June 24-27, 1998.

Received for publication July 15, 1998; revisions requested Sept 4, 1998; revisions received Feb 10, 1999; accepted for publication March 8, 1999.

Address for reprints: Curtis G. Tribble, MD, Department of Surgery, Division of Thoracic and Cardiovascular Surgery, Box 3111, MR4 Building, University of Virginia Health Sciences Center, Charlottesville, VA 22908.

Copyright $\odot 1999$ by Mosby, Inc.

$0022-5223 / 99 \$ 8.00+0 \quad \mathbf{1 2 / 6 / 9 8 4 3 2}$ in resection of these aneurysms. The incidence of spinal cord dysfunction varies, but the largest series to date reported the development of postoperative paraplegia or paraparesis in $16 \%$ of their patients. ${ }^{1}$ Spinal cord injury during the repair of thoracoabdominal aneurysms is the result of ischemia caused by aortic crossclamping and ischemia caused by devascularization from interruption of the critical blood supply of the spinal cord. However, owing to the variability and complexity of the blood supply of the spinal cord, the management of this critical circulation remains controversial. The most important artery of the thoracolumbar region of the spinal cord may be the artery of Adamkiewicz, arising most commonly in the region from the ninth thoracic intercostal to the first lumbar artery. The reattachment of these intercostal and lumbar arteries when replacing thoracoabdominal aneurysms has been postulated to preserve the artery of Adamkiewicz and play an important role in preserving spinal cord function. ${ }^{2-8}$ However, 
Table I. Preoperative patient characteristics

\begin{tabular}{lllc}
\hline Characteristic & \multicolumn{1}{c}{$\begin{array}{c}\text { Group I } \\
(n=91)\end{array}$} & $\begin{array}{l}\text { Group II } \\
(n=41)\end{array}$ & P value \\
\hline Age (y) & 60.8 & 65.5 & .29 \\
Male (\%) & $52 / 91(57.1)$ & $27 / 41(65.9)$ & .34 \\
Hypertension (\%) & $61 / 91(67.0)$ & $32 / 41(78.0)$ & .20 \\
Myocardial infarction & $20 / 91(22.0)$ & $12 / 41(29.3)$ & .37 \\
$\quad(\%)$ & & & \\
Prior aortic surgery & $26 / 91(28.6)$ & $12 / 41(29.3)$ & .93 \\
$\quad(\%)$ & & & \\
Aneurysm extent & & & \\
$\quad$ Type I (\%) & $53 / 91(58.2)$ & $21 / 41(51.2)$ & \\
$\quad$ Type II (\%) & $14 / 91(15.4)$ & $6 / 41(14.6)$ & \\
$\quad$ Type III (\%) & $10 / 91(11.0)$ & $5 / 41(12.2)$ & \\
$\quad$ Type IV (\%) & $14 / 91(15.4)$ & $9 / 41(22.0)$ & \\
Dissection & & & .47 \\
$\quad$ Chronic (\%) & $15 / 91(16.5)$ & $5 / 41(12.2)$ & \\
$\quad$ Acute (\%) & $9 / 91(9.9)$ & $2 / 41(4.9)$ & \\
Rupture (\%) & $18 / 91(19.8)$ & $3 / 41(7.3)$ & .07 \\
\hline
\end{tabular}

others have questioned the significance of reimplanting these vessels for spinal protection. ${ }^{9}$

$\mathrm{We}^{10}$ have previously reported our experience with the clamp-and-sew technique for the repair of thoracoabdominal aortic aneurysms. Recently, we have modified our technique of these repairs by preserving all patent vessels between the eighth thoracic intercostal and the second lumbar arteries in an effort to reduce the incidence of spinal cord ischemia. The current study examines the addition of intercostal artery reimplantation to the clamp-and-sew technique and its influence on postoperative paraplegia without additional combinations of adjuvant techniques for spinal cord protection.

\section{Patients and methods}

Patient population. All patients undergoing clamp-andsew repair of aneurysms of the thoracoabdominal aorta at the University of Virginia Health Sciences Center from January 1987 through December 1997 were included in the study. A total of 132 patients were identified for review. Ninety-one patients in group I underwent aneurysm repairs before January 1995 and did not undergo intercostal artery reimplantation. In these patients, potentially critical intercostal vessels were oversewn. Group II included 41 patients who underwent aneurysm operations from January 1995 through December 1997 and had critical vessels between the eighth thoracic intercostal and the second lumbar arteries reimplanted to the graft or preserved at the aortic anastomoses. Patients with isolated aneurysms of the descending thoracic aorta were not included in this study. Patients who underwent repair with the use of cardiopulmonary bypass, left atrial-femoral artery bypass, or hypothermic circulatory arrest were excluded. During the more recent era of the study since January 1995, 6 patients required surgical repair by means of these various techniques. These techniques were generally applied in cases in which the proximal aorta was not able to be clamped or in cases in which there was preoperative evidence of severe left ventricular dysfunction.

Medical records of all patients were reviewed and preoperative variables of age, sex, thoracoabdominal aneurysm extent, symptomatic status, the presence of dissection, the presence of rupture, history of hypertension, history of myocardial infarction or congestive heart failure, and previous aortic resection were obtained. Thoracoabdominal aneurysm extent was classified according to the scheme proposed by Crawford: type I, entire descending and upper abdominal aorta; type II, entire descending and abdominal aorta; type III, variable extents of descending and abdominal aorta; and type IV, entire abdominal aorta with the graft extending proximal to the celiac axis. The aortic segment that was resected was used to determine the classification of aneurysm extent. The duration of aortic occlusion was obtained from the operative record for each patient. The postoperative outcomes of death and spinal cord injury manifested by paraplegia or paraparesis were determined for each patient. Operative mortality included deaths within the patients' entire hospital length of stay or within 30 days after the operation if they were discharged from the hospital. All patients who died after the operation had thorough evaluations for neurologic injury recorded before death. We defined paraplegia as a permanent neurologic deficit and paraparesis as temporary weakness of a lower extremity.

The two groups of patients were not significantly different with respect to a number of preoperative characteristics that are believed to have an impact on outcome in patients with aneurysmal disease, as shown in Table I. Specifically, the extent of the aortic lesions did not differ significantly between the two groups of patients. In particular, $66 \%$ of the more recent group and $73 \%$ of the earlier group of patients had thoracoabdominal aneurysms classified as Crawford type I and type II aneurysms, indicating that a majority of patients in each group had lesions posing a relatively high risk for paraplegia. In addition, we used a multivariable logistic regression analysis to create a propensity score for each patient in the data set. In the analysis, we found the logistic models with and without the propensity scores as a covariate to have almost identical estimates of the baseline characteristics of the two patient groups.

Operative technique. All thoracoabdominal repairs were carried out completely retroperitoneally through a thoracoabdominal incision. All patients were intubated with a doublelumen endotracheal tube that permitted deflation of the left lung, facilitating exposure and repair. Intraoperatively, arterial blood pressure, central venous pressure, pulmonary artery pressure, continuous electrocardiogram, urine output, temperature, arterial blood gases, electrolytes, and hemoglobin were monitored. No active systemic or regional hypothermia was used. Before aortic clamping, afterload reduction was achieved with sodium nitroprusside. In addition, patients 
Table II. Influence of preoperative and intraoperative risk factors on operative mortality

\begin{tabular}{|c|c|c|c|}
\hline \multirow[b]{2}{*}{ Risk factor } & \multicolumn{2}{|c|}{ Operative death } & \multirow[b]{2}{*}{$\mathrm{P}$ value } \\
\hline & $\begin{array}{c}Y e s(n=14) \\
{[n(\%)]}\end{array}$ & $\begin{array}{c}\text { No }(n=118) \\
{[n(\%)]}\end{array}$ & \\
\hline Age $>70 y$ & $6 / 14(42.9)$ & $38 / 118(32.2)$ & .42 \\
\hline TAA type I or II & $12 / 14(85.7)$ & $75 / 118$ (63.6) & .09 \\
\hline Dissection & 4/14 (28.6) & 27/118 (22.9) & .74 \\
\hline Rupture & $5 / 14(35.7)$ & $16 / 118(13.6)$ & .05 \\
\hline $\begin{array}{l}\text { Aortic clamp time } \\
>30 \mathrm{~min}\end{array}$ & $6 / 14(42.9)$ & $47 / 118(39.8)$ & .83 \\
\hline $\begin{array}{l}\text { Critical intercostal } \\
\text { sacrifice }\end{array}$ & $12 / 14(85.7)$ & 79/118 (66.9) & .22 \\
\hline
\end{tabular}

TAA, Thoracoabdominal aneurysm.

received a 100 unit $/ \mathrm{kg}$ dose of heparin intravenously, as well as a bolus of intravenous mannitol and furosemide. No pharmacologic pretreatment with calcium channel blockers, steroids, or opiate antagonists was used in any case.

The clamp-and-sew technique of aneurysm resection and repair was used in all operations. ${ }^{3-5,10-13}$ The double crossclamp technique was used most frequently, but anatomic concerns occasionally necessitated repair with a single proximal crossclamp and controlled distal exsanguination. Since January 1995, patent segmental intercostal arteries and lumbar arteries between the eighth thoracic intercostal and second lumbar vessels have been preserved or reconstructed in all patients. No attempt was made to preoperatively identify the artery of Adamkiewicz with angiography. In addition, no adjunctive technique for spinal cord monitoring, including somatosensory-evoked potentials, ${ }^{9}$ was used to intraoperatively identify critical intercostal or lumbar arteries that supplied the spinal cord. During the more recent era, the intercostal arteries were preserved by excising an oval from the graft and reattaching the critical intercostal arteries to the aortic graft as a Carrel patch in 12 patients $(29.3 \%)$, or by using a beveled aortic anastomosis at the proximal or distal end to include the critical intercostal and lumbar arteries in 29 patients $(70.7 \%)$. For all patent intercostal and lumbar arteries, preservation was carefully planned with either a Carrel patch or a beveled anastomosis, but the patch or bevel was not extended to T8 or T9 if there was no visibly patent intercostal artery at that level. No patient in this study underwent distal aortic perfusion or cerebrospinal fluid drainage.

Statistical methods. Data were analyzed by the statistics program SPSS (SPSS Inc, Chicago, Ill). Categoric variables were analyzed by $\chi^{2}$ analysis and Fisher's exact test. Fisher's exact test was used for $2 \times 2$ tables when the assumptions for $\chi^{2}$ were not satisfied. $\chi^{2}$ Analysis was used in Table I, and Fisher's exact test was used in Tables II and III. The numeric variable of aortic clamp time is reported as mean \pm standard deviation and was analyzed by means of the Student $t$ test. A multivariable logistic regression that included date of operation, age of the patient at operation, aortic clamp time, aneurysm extent, presence of aortic dissection, presence of aor-
Table III. Influence of preoperative and intraoperative risk factors on postoperative paraplegia/paraparesis

\begin{tabular}{lccc}
\hline & \multicolumn{2}{c}{ Paraplegia/paraparesis } & \\
\cline { 2 - 3 } & $\begin{array}{c}\text { Yes }(n=10) \\
{[n(\%)]}\end{array}$ & $\begin{array}{c}\text { No }(N=122) \\
{[n(\%)]}\end{array}$ & P value \\
\hline Risk factor & $4 / 10(40)$ & $40 / 122(32.8)$ & .73 \\
Age > 70 y & $4 / 10(40)$ & $83 / 122(68.0)$ & .09 \\
TAA type I or II & $2 / 10(20)$ & $29 / 122(23.8)$ & 1.00 \\
Dissection & $2 / 10(20)$ & $19 / 122(15.6)$ & .66 \\
Rupture & $3 / 10(30)$ & $35 / 122(28.7)$ & 1.00 \\
Prior aortic surgery & $9 / 10(90)$ & $82 / 122(67.2)$ & .17 \\
$\begin{array}{l}\text { Critical intercostal } \\
\quad \text { sacrifice }\end{array}$ & & & \\
Aortic clamp time & $30.6 \pm 7.1$ & $30.5 \pm 14.7$ & .98 \\
$\quad$ (min) & & & \\
\hline
\end{tabular}

TAA, Thoracoabdominal aneurysm.

tic rupture, and history of prior aortic surgery was performed to identify the variables associated with postoperative neurologic injury. This retrospective review of our evolving experience with the new technique of intercostal preservation and reimplantation raises the possibility of a statistical type I error, concluding a difference when no true difference exists. We therefore explicitly state all actual $P$ values for interpretation.

The propensity score is the conditional probability of assignment to a particular group given an individual's set of baseline descriptors. Propensity scores can be used to reduce bias that is attributed to the confounding effect of having large differences in the magnitude of baseline values observed within two groups. A multivariable logistic regression analysis was used to create a propensity score for each patient in the data set, which represents the patient's probability of belonging to the more recent group when the probability is conditioned on the patient's set of baseline values. The propensity score analysis was done by using the program S-Plus version 4.5 (Mathsoft, Inc, Seattle, Wash).

\section{Results}

The operative mortality rate was $13.2 \%$ (12/91) in the earlier group (group I), and this rate decreased to $4.9 \%$ $(2 / 41)$ in the more recent group of patients (group II) $(P$ $=.22$ ). The influence of specific preoperative and intraoperative variables on the incidence of death is shown in Table II. Not surprisingly, the clinical circumstance of aortic aneurysm rupture was significantly associated with mortality. In addition, the more extensive thoracoabdominal aneurysms had a strong influence on the incidence of death, but this association did not reach statistical significance.

The incidence of postoperative paraplegia was significantly lower in the more recent cohort of patients ( $8.8 \%$ in group I vs $0 \%$ in group II, $P=.05 ; 95 \%$ con- 


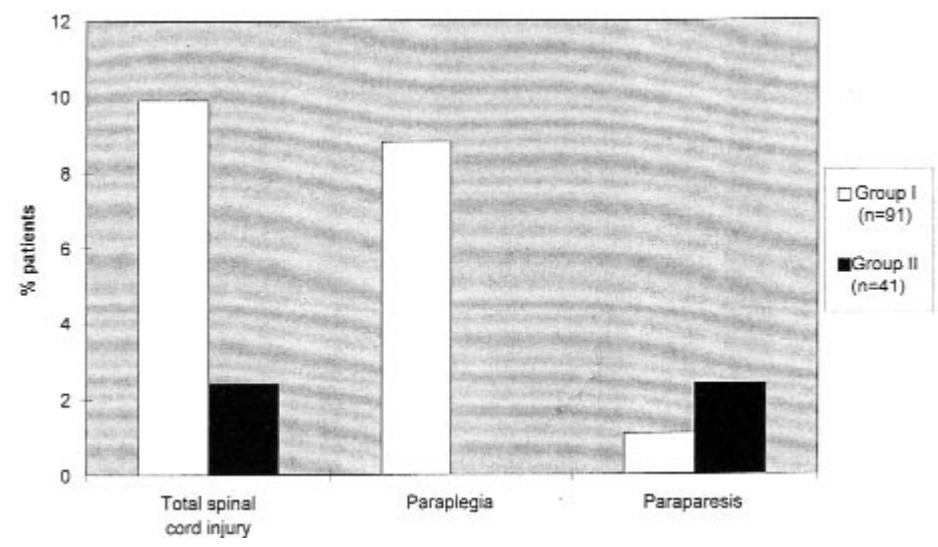

Fig 1. Comparison between group I and group II patients with respect to spinal cord injury after aneurysm resection. With intercostal artery reimplantation, the more recent cohort of patients had a lower overall incidence of spinal cord injury (9/91 [9.9\%] in group I vs $1 / 41[2.4 \%]$ in group II; $P=.17$; $95 \%$ CI for the difference of proportions, $-0.0029,0.1519)$ and a significantly lower rate of paraplegia $(8 / 91[8.8 \%]$ in group I vs $0 / 41[0 \%]$ in group II; $P=.05 ; 95 \% \mathrm{CI}$ for the difference of proportions, $(0.0297,0.1461)$. The incidence of paraparesis was not different between the groups.

fidence interval $[\mathrm{CI}]$ for the difference of proportions, $0.0297,0.1461$ ) (Fig 1). The overall rate of spinal cord dysfunction was lowered from $9.9 \%$ (9/91) in the earlier group to $2.4 \%(1 / 41)$ in the more recent group of patients $(P=.17 ; 95 \% \mathrm{CI}$ for the difference of proportions, $-0.0029,0.1519)$. One patient operated on since the institution of intercostal artery reimplantation had lower extremity paraparesis, defined as weakness with preservation of motion against gravity or resistance. This particular patient had a type III thoracoabdominal aneurysm that was symptomatic but had no evidence of dissection or rupture. A beveled proximal anastomosis was constructed to preserve patent intercostal arteries. The celiac artery, superior mesenteric artery, and right renal artery were then reimplanted as a separate Carrel patch. The distal anastomosis was then completed, the clamps were removed, and the left renal artery was reimplanted with the aid of a partial-occlusion clamp. Aortic crossclamp time was only 26 minutes, and there were no significant times of hemodynamic instability during the perioperative or intraoperative periods. This patient fortunately recovered full neurologic function of the lower extremities. Analysis of variables examined for their relation to postoperative neurologic deficit is displayed in Table III. A multivariable logistic regression analysis identified only aneurysm extent (Crawford type I and type II) as a predictor of less postoperative spinal cord injury $(P=.08)$. No other risk factor, including date of operation, age of patient at operation, aortic clamp time, aortic dissection, aortic rupture, or prior aortic surgery, was significantly asso- ciated with the incidence of postoperative spinal cord ischemic complications.

The average aortic crossclamp time in group I was $30.3 \pm 11.5$ (SD) minutes (range 12-95 minutes). With the institution of intercostal reimplantation in the more recent group of patients, the time of aortic occlusion was not significantly prolonged, with an average crossclamp time of $31.0 \pm 21.0(\mathrm{SD})$ minutes (range 15-110 minutes) $(P=.88)$. The majority of patients in group I $(53 / 91$ [58.2\%]) and the majority of patients in group II (26/41 [63.4\%]) underwent thoracoabdominal aortic aneurysm repair with clamp times of 30 minutes or less. Overall, the combined incidence of mortality and spinal cord injury decreased from $23.1 \%$ (21/91) to $7.3 \%(3 / 41)(P=.03)$, and the time of aortic occlusion was not lengthened with the addition of intercostal artery preservation.

\section{Discussion}

The improvement in the results of operations for thoracoabdominal aortic aneurysms seen in our most recent group of patients shows that significant progress is being made in the management of these challenging lesions. The favorable outcomes of the recent operations are likely due to the specific modifications to the operative procedure, as well as the improvements in anesthesia and nursing care over time. This raises the possibility of a bias in favor of improved outcomes in any recent group of patients when compared with a group of historical controls. However, in an experience spanning 29 years, Lawrie, Earle, and DeBakey ${ }^{12}$ found that the 
incidence of paraplegia remained unchanged over time in all patients who underwent technically similar surgical repair of thoracoabdominal aneurysms. This experience highlights the critical influence of surgical technique on the postoperative complication of spinal cord ischemia and permits our investigation of a surgical modification over time and its influence on postoperative paraplegia. In addition, there were no significant preoperative differences between the two groups of patients that may have influenced the postoperative neurologic outcome, and propensity scores described almost identical estimates of the two groups. Logistic regression analysis found only the Crawford type I and type II thoracoabdominal aneurysms to be associated with the incidence of paraplegia or paraparesis, and the extent of aneurysms was similar between the two groups. Although not statistically significant, the more recent group included fewer patients who had aortic rupture on presentation. Inasmuch as rupture was significantly associated with the incidence of mortality, a lower prevalence of rupture may have contributed to the lower mortality in the more recent group. However, aneurysmal rupture did not correlate with neurologic injury and was therefore less likely to influence the reduced incidence of paraplegia.

During our 10-year experience, we have continued to be strong supporters of the clamp-and-sew technique of surgical repair of thoracoabdominal aneurysms. We $\mathrm{We}^{10}$ and others ${ }^{3-7,11-14}$ have reported low rates of ischemic spinal cord complications with the clamp-and-sew method, but the incidence of this devastating complication has not reached zero. In an effort to further decrease the incidence of postoperative paraplegia, we added the reimplantation of all patent intercostal and lumbar vessels in the region of the eighth thoracic intercostal to the second lumbar arteries to our surgical repairs.

The cause of neurologic deficits is believed by many to be multifactorial, but the two principal reasons for spinal cord ischemia during thoracoabdominal aneurysm repair are the temporary aortic occlusion during crossclamping and the permanent interruption of critical radiculomedullary feeder vessels to the spinal cord. Therefore, it is intuitively logical to attempt to minimize overall clamp time and to reattach the blood supply to the spinal cord. Aortic crossclamp time has been shown to be an independent predictor of paraplegia or paraparesis, ${ }^{1}$ and crossclamp time less than 30 minutes has been shown to be an independent factor predictive of the absence of paraplegia. ${ }^{12}$ In addition, regardless of the operative spinal cord ischemic period, the permanent interruption of critical intercostal arteries, in patients in whom they provide the direct arterial circu- lation to the spinal cord, can produce spinal cord infarction. Experimentally, the influence of sacrificing intercostal and lumbar vessels, particularly in the region of the eleventh thoracic intercostal to the first lumbar arteries, on the incidence of postoperative paraplegia has been confirmed. ${ }^{14,15}$ Clinically, Svensson and associates ${ }^{7}$ reported that $48 \%$ of patients who had one or more arteries between the eleventh thoracic intercostal and the first lumbar vessels oversewn had neurologic deficit, compared with $27 \%$ of patients who did not have patent arteries or who had all patent arteries reattached in this region. Griepp and colleagues ${ }^{9}$ showed that the risk of paraplegia was increased 29 times in cases in which greater than 10 intercostal arteries were sacrificed.

These findings correlate with our understanding of the blood supply to the spinal cord. The anterior longitudinal spinal artery provides the blood supply to the anterior two thirds of the spinal cord and is a vascular trunk composed of branches from the anterior radiculomedullary arteries. These radiculomedullary arteries arise from the segmental spinal arteries, which in turn arise from the intercostal and lumbar arteries. The artery of Adamkiewicz is described as the largest of these radiculomedullary arteries and most commonly arises between the ninth thoracic intercostal and the first lumbar vessels. ${ }^{2}$ However, recent evidence questions the existence of a single artery of Adamkiewicz and proposes a model of multiple inputs to the anterior spinal artery. ${ }^{9}$ In addition, the continuity of the anterior spinal artery cannot be relied on, as it is now described as a network of interconnected vascular anastomoses. ${ }^{8}$ In this model, areas between major segments of the anterior spinal arterial plexus may be only poorly interconnected and would be at increased risk for ischemic damage with any alteration of the blood supply. Thus both the findings of prior studies and the anatomic blood supply of the spinal cord lend credence to the preservation and reimplantation of intercostal arteries in the segment from the eighth thoracic intercostal to the second lumbar vessels.

In our technique for repair of thoracoabdominal aneurysms, we support the "blinded" reattachment of all patent vessels between the eighth thoracic intercostal and the second lumbar arteries. Kieffer, ${ }^{2}$ Heinemann, ${ }^{8}$ and their associates are supporters of preoperative angiography for the localization of the artery of Adamkiewicz and other vessels that supply the spinal cord to plan their reattachment at the time of surgery. However, the yield of complete identification has been only approximately $65 \%$, and it has been even more difficult, if not impossible, to find those impor- 
tant collateral vessels with angiography. The invasive and often time-consuming technique of selective angiography has also been shown to have a $4.6 \%$ incidence of angiography-related complications. ${ }^{8}$ Several authors ${ }^{6,9,16}$ are proponents of monitoring spinal cord function with somatosensory-evoked potentials to identify critical aortic segments that supply the spinal cord. However, there is an approximately 5\% incidence of false negatives, and one study found that no relationship could be demonstrated between the extinction time, the recovery time, or the duration of loss of evoked potentials with postoperative neurologic outcome. ${ }^{16}$ Since somatosensory evoked potentials observe mainly the posterior spinal columns, which are not supplied primarily by the artery of Adamkiewicz, it has been suggested that the recording of motor-evoked myogenic potentials would be a more accurate intraoperative monitoring technique. ${ }^{17}$ This technique is elaborate, time-consuming, and requires significant neurologic expertise, which renders it more of a research tool at present.

Our $2.4 \%$ incidence of spinal cord injury with the institution of intercostal artery preservation compares favorably with the results of contemporary reports supporting similar practices $3,4,7$ and with those that use varying combinations of methods for spinal cord protection. ${ }^{9,16}$ A critical point in the current study is that these improved results occurred with intercostal artery reimplantation as the only adjuvant technique for spinal cord protection during clamp-and-sew repair, inasmuch as no patient underwent left heart bypass, cerebrospinal fluid drainage, or pharmacologic intervention. Although other effective methods for spinal cord protection during thoracoabdominal aneurysm repair do exist, our isolated investigation at one institution of the efficacy of intercostal artery preservation confirms the beneficial role of thoracic intercostal and lumbar artery reimplantation on lowering the incidence of paraplegia. In addition, our findings support that this technique for spinal cord protection can be added to the expeditious clamp-and-sew repair without significant prolongation of aortic crossclamp time. This is likely the result of two factors. First, training in this operation has significantly improved over the years, and this clearly plays a role in the clamp times in the more recent cohort of patients. Second, extensive beveling of the aortic graft to include the intercostal and lumbar arteries at the anastomoses has also been a factor in minimizing aortic clamp times while still preserving the critical blood supply to the spinal cord.

The sacrifice of the direct spinal cord blood supply places patients in a precarious anatomic position in which their cords are largely dependent only on collateral circulation. This position largely increases the likelihood of spinal cord ischemia and the subsequent development of postoperative paraplegia or paraparesis, particularly during periods of perioperative hemodynamic instability. With the variability of the blood supply to the spinal cord and the difficulties inherent in the methods developed to identify the critical circulation, we support the expeditious clamp-and-sew surgical repair of thoracoabdominal aneurysms with the preservation of all patent feeder vessels between the eighth thoracic intercostal and second lumbar arteries to avoid placing patients in such an unstable position. Although this practice has produced extremely low rates of spinal cord complications at our institution, it may not be universally reproducible and it has not guaranteed the complete absence of postoperative paraplegia. This highlights the need for continued research in developing effective strategies for complete spinal cord protection during the surgical repair of thoracoabdominal aneurysms.

\section{REFERENCES}

1. Svensson LG, Crawford ES, Hess KR, Coselli JS, Safi HJ. Experience with 1509 patients undergoing thoracoabdominal aortic operations. J Vasc Surg 1993;17:357-70.

2. Kieffer E, Richard T, Chiras J, Godet G, Cormier E. Preoperative spinal cord arteriography in aneurysmal disease of the descending thoracic and thoracoabdominal aorta: preliminary results in 45 patients. Ann Vasc Surg 1989;3:34-46.

3. Cambria RP, Davison JK, Zannetti S, L'Italien G, Atamian S. Thoracoabdominal aneurysm repair: perspectives over a decade with the clamp-and-sew technique. Ann Surg 1997;226:294-305.

4. Coselli JS. Thoracoabdominal aortic aneurysms: experience with 372 patients. J Card Surg 1994;9:638-47.

5. Coselli JS, Plestis KA, LaFrancesca S, Cohen S. Results of contemporary surgical treatment of descending thoracic aortic aneurysms: experience in 198 patients. Ann Vasc Surg 1996;10: 131-7.

6. Grabitz K, Sandmann W, Stuhmeier K, Mainzer B, Godehardt E, Ohle B, et al. The risk of ischemic spinal cord injury in patients undergoing graft replacement for thoracoabdominal aortic aneurysms. J Vasc Surg 1996;23:230-40.

7. Svensson LG, Hess KR, Coselli JS, Safi HJ. Influence of segmental arteries, extent, and atriofemoral bypass on postoperative paraplegia after thoracoabdominal aortic operations. J Vasc Surg 1994;20:255-62

8. Heinemann MK, Brassel F, Herzog T, Dresler C, Becker H, Borst HG. The role of spinal angiography in operations on the thoracic aorta: Myth or reality? Ann Thorac Surg 1998;65:346-51.

9. Griepp RB, Ergin MA, Galla JD, Lansman S, Khan N, Quintana $\mathrm{C}$, et al. Looking for the artery of Adamkiewicz: a quest to minimize paraplegia after operations for aneurysms of the descending thoracic and thoracoabdominal aorta. J Thorac Cardiovasc Surg 1996;112:1202-15.

10. Mauney MC, Tribble CG, Cope JT, Tribble RW, Spotnitz WD, Kron IL. Is clamp and sew still viable for thoracic aortic resection? Ann Surg 1996;223:534-43. 
11. Cooley DA. Single-clamp repair of aneurysms of the descending thoracic aorta. Semin Thorac Cardiovasc Surg 1998;10:87-90.

12. Lawrie GM, Earle N, DeBakey ME. Evolution of surgical techniques for aneurysms of the descending thoracic aorta: twentynine years' experience with 659 patients. J Card Surg 1994;9:648-61.

13. Scheinin SA, Cooley DA. Graft replacement of the descending thoracic aorta: results of "open" distal anastomosis. Ann Thorac Surg 1994;58:19-23.

14. Svensson LG, Patel V, Robinson MF, Ueda T, Roehm JOJ, Crawford ES. Influence of preservation or perfusion of intraoperatively identified spinal cord blood supply on spinal motor evoked potentials and paraplegia after aortic surgery. J Vasc Surg 1991;13:355-65.

15. Dapunt OE, Midulla PS, Sadeghi AM, Mezrow CK, Wolfe D, Gandsas A, et al. Pathogenesis of spinal cord injury during simulated aneurysm repair in a chronic animal model. Ann Thorac Surg 1994;58:689-97.

16. Schepens MA, Boezeman EH, Hamerlijnck RP, terBeek H, Vermeulen FE. Somatosensory evoked potentials during exclusion and reperfusion of critical aortic segments in thoracoabdominal aortic aneurysm surgery. J Card Surg 1994;9:692-702.

17. DeHaan P, Kalkman CJ, deMol BA, Ubags LH, Veldman DJ, Jacobs MJHM. Efficacy of transcranial motor-evoked myogenic potentials to detect spinal cord ischemia during operations for thoracoabdominal aneurysms. J Thorac Cardiovasc Surg 1997;113:87-101.

\section{Discussion}

Dr D. Craig Miller (Stanford, Calif). Dr Ross, you have done a splendid job. However, I have a lot of difficulty in accepting your cause-and-effect conclusions.

On the surface, what this paper is asking us to do is accept the premise that being more aggressive about reimplanting some intercostal arteries has resulted directly in the mortality rate falling from $13 \%$ to $5 \%$ and a decrease in the spinal cord injury rate from $10 \%$ to $2.4 \%$, in what I submit is a relatively low-risk patient population for spinal cord injury. For example, only 26 of your 132 patients had aortic dissections, who historically have been at highest risk, which indicates that a very large majority of your patients did not have dissections; given your age range of 60 to 66 years, most probably had aneurysms of atherosclerotic origin, where patency of many critical intercostal arteries is dubious preoperatively owing to the laminated thrombus that is present in these aneurysms. This is exemplified by looking at your paraplegia data: In the 10 patients who were totally paralyzed, critical intercostal arteries had been sacrificed in $90 \%$, but they were sacrificed in $67 \%$ of your patients who did not have paraplegia. I am having trouble accepting that the purported improved results are directly due to your change in technique.

Before we get to this, you have honestly stated that this is a selected series. You excluded what I would consider to be a higher risk subset of patients in whom you had to use cardiopulmonary bypass with or without profound hypothermic circulatory arrest (or left atrial-femoral artery bypass), which is the way many of us perform most operations for descending thoracic or thoracoabdominal aortic aneurysms. Indeed, one of the most important things we have learned over the past decade is to avoid clamping the proximal descending aorta or distal transverse arch whenever possible, as advocated by Nicholas Kouchoukos. My question is this: How many patients did you exclude from this analysis between 1987 and 1997 and what were the spinal cord injury and death rates in that other group that you did not tell us about today?

Dr Ross. Over the past 3 years of our experience, only about 1 to 2 operations per year have been performed with the use of cardiopulmonary bypass, left atrial-femoral artery bypass, or hypothermic circulatory arrest. It is important to emphasize that at the University of Virginia we only use those bypass techniques if the clamp-and-sew repair is impossible. As you said, that mainly includes patients with such a highly diseased aorta that the proximal clamp cannot be placed. In our institution, it is a very small group and it is a select group that has a whole host of other risk factors. In this small, select, high-risk group, our mortality is justifiably higher and approaches approximately 25\%. In this small group, our paraplegia rate has not been different, but that is a small number of patients. I must emphasize, however, that it is important that we perform this clamp-and-sew technique if at all possible, and that is why we focused our study on just that group.

Dr Miller. If that means 5 to 8 cases a year times 10 years, it would not be such a small cohort of patients. That is almost as big as the more recent population you are telling us about today.

Those of us who have watched Irving Kron operate know he is a very slick, very fast, left-handed surgeon and loves that big 2-0 Prolene suture on that giant MH needle. He really whips these cases through. I think that explains the very short crossclamp times in this series. Dr Kron is one of the few remaining Denton Cooley disciples left who do not resort to some sort of cardiopulmonary bypass perfusion very often.

Nevertheless, even in Dr Kron's hands, I do not believe your group could have reimplanted many pairs of critical intercostal arteries as separate Carrel buttons during the 30 or so minutes of crossclamp time, especially in the Crawford type II cases, where you also need time to sew in a button for the visceral and the renal arteries. I suspect you are using a very long, beveled distal anastomosis for the Crawford type I thoracoabdominal aortic aneurysm cases; for the type IIIs and IVS, you probably are just doing more elongated beveled proximal anastomoses. My question is how many patients had separate Carrel patches of intercostal arteries reimplanted in your most recent cohort.

Dr Ross. That is correct. The focus of our study was preservation of the intercostal arteries. In the more recent group, $66 \%$ of our patients had the type I and type II aneurysms. But of these aneurysms, only a small number, approximately $15 \%$ of the total, were type IIs, which would then lead to having a separate Carrel patch. The majority of type I aneurysms have more of a beveled anastomosis. Since 1995, we very diligently have planned things before the clamp has gone on, to dissect things out and actually plan 
how to preserve the intercostal arteries. But patients with type II aneurysms, even though that was a lower number, did get separate Carrel patches, and that led to a numbers game with a skew toward an average that was approximately similar, but included clamp times up to as high as 50 to 60 minutes.

Dr Miller. Perhaps I should point out that in your manuscript you do state that you frequently use Cooley's methods, that is, no distal clamp and partial exsanguination. This feature-avoiding a distal clamp—is very advantageous; we do that a lot at Stanford.

My major qualm with your data is that you did not do a multivariable statistical analysis. We all know that over time everything usually gets better in cardiovascular surgery. However, changes in your patient population and patient selection criteria might explain the reduction in morbidity and mortality more than just this more aggressive use of long, beveled anastomoses. For example, the proportion of nondissectors went from $73 \%$ to $82 \%$ between the two groups. The incidence of rupture between the two groups fell from $20 \%$ earlier to $7 \%$ more recently. The fraction of patients who had Crawford type I or II aneurysms fell from $74 \%$ to $66 \%$. I do admit that none of these differences was significant in your univariate statistics. However, could entering all these data, including year of operation, into a multivariable model have resulted in rejection of your null hypothesis? Put another way, could this have been one of those true, true, but unrelated situations? Although strictly speaking it is impossible to prove cause and effect in any retrospective analysis using historical controls, I am not certain we honestly should take your bottom line message home and believe that reimplanting more intercostal arteries really has any bearing on the incidence of paraplegia and paraparesis without at least some type of multivariable analysis. Indeed, even had you not changed your surgical strategy recently, you might have seen the same results over time.

Dr Ross. I think that the question of time using historical controls is a very good one, and we did not do a multivariate analysis. We point a little bit to the largest series to date of Dr Stanley Crawford, who also used historical controls in his series, and he reported that there was no difference in paraplegia rates based on surgical technique over a 30-year span. In addition, as you said, the two groups that we used were not significantly different. There was a trend toward a fewer number of ruptures in our most recent group of patients, and that clearly has a role in the reduction of mortality in that group of patients. However, no variable was found to significantly predict postoperative spinal cord ischemic complications. In our analysis, if we eliminated the ruptures from both groups, the incidence of paraplegia was still significantly lower in our most recent group.

Dr Miller. You are a surgical resident at a fine university medical center, and I am sure you have heard your mentors say that the best predictor of results is patient selection, patient selection, patient selection, and even more patient selection. Maybe the attendings at the University of Virginia have gotten a bit wiser over the last two decades concerning whom they operate on and which techniques they use for which individual patients. 